\title{
Identification and Genetic Diversity of Formae Speciales of Setosphaeria turcica in China
}

L. Tang, Plant Protection College, Shenyang Agricultural University, Shenyang, China 110866, and Life Science College, Luoyang Normal University, Luoyang, China 471000; and Z. G. Gao and Y. Yao, Plant Protection College, and X. Liu, College of Biosciences and Technology, Shenyang Agricultural University

\begin{abstract}
Tang, L., Gao, Z. G., Yao, Y., and Liu, X. 2015. Identification and genetic diversity of formae speciales of Setosphaeria turcica in China. Plant Dis. 99: $482-487$.

Setosphaeria turcica is an important pathogen that causes northern leaf blight of corn and sorghum. In total, 188 isolates were isolated from diseased leaves of maize and sorghum in China. However, differences in morphology were not apparent among $S$. turcica isolates derived from different hosts. The formae speciales were identified as S. turcica. f. sp. zeae and S. turcica. f. sp. sorghi. Universally primed polymerase chain reaction (UP-PCR) analysis led to the conclusion that genetic differences were

present among these formae speciales and divided these isolates into five clades. The isolates of each clade belonged to the same forma specialis, and different formae speciales were assigned to different clades. The results of the present study demonstrated that pathogenic specialization of the $S$. turcica isolate correlated more closely with genetic diversity than geographical distribution. Therefore, combined with assays for pathogenicity, UP-PCR analysis yields an enhanced understanding of the formae speciales of $S$. turcica
\end{abstract}

Northern leaf blight of corn and sorghum caused by Setosphaeria turcica (Luttr.) K. J. Leonard \& Suggs (21) is one of the important foliar diseases of corn and sorghum wherever they are cultivated (13). Historically, isolates of S. turcica were divided into formae speciales according to their virulence for a particular host or group of hosts. Since Mitra (26) first reported specialization of S. turcica on maize or sorghum, Johnson grass and Sudan grass were identified as hosts as well $(3,4,20,21,25,29,30)$. Therefore, $S$. turcica is classified into the following types: S. turcica. f. sp. zeae, S. turcica. f. sp. sorghi, and $S$. turcica. f. sp. complexa. S. turcica.f. sp. zeae is only virulent on maize, $S$. turcica. f. sp. sorghi infects sorghum and Sudan grass, and $S$. turcica. f. sp. complexa infects each of the hosts listed here. Therefore, two formae speciales of $S$. turcica, $S$. turcica f. sp. zeae and $S$. turcica f. sp. sorghi, only infect specific compatible hosts and induce typical spots. S. turcica. f. sp. complexa also is called a nonforma specialis (1).

Previous research on the formae speciales of $S$. turcica was limited to pathogenicity tests of host-pathogen interactions; however, to our knowledge, there are no studies of the molecular mechanism and genetic basis of pathogenic specialization of S. turcica in China. Moreover, determining pathogenicity by analyzing host-pathogen interactions is subjective and inaccurate and, therefore, not comprehensive. For example, molecular biology techniques are widely used to explore genetic variability of fungi $(10,15,36-38)$. Therefore, analyzing the genetic diversity of pathogens represents an important aspect of the comprehensive analysis of formae speciales (37).

To our knowledge, there are no published studies of the genetic relationships among formae speciales of $S$. turcica. Universally primed polymerase chain reaction (UP-PCR) is a fingerprinting method that amplifies the DNA of any organism without previous knowledge of DNA sequences and generates multiband profiles (fingerprints) using gel electrophoresis $(7-9,23,28)$. UP-PCR primers can be designed to amplify the intergenic variable regions of genomes (6). For example, the UP-PCR technique was applied successfully to characterize the genomes of pathogens such as Fusarium spp., Rhizoctonia solani, Trichoderma spp., Gliocladium spp., and Pyrenophora graminea $(7,15,23)$.

Corresponding author: Z. G. Gao; E-mail: gaozenggui@ sina.com

Accepted for publication 19 November 2014.

http://dx.doi.org/10.1094/PDIS-06-14-0570-RE

(C) 2015 The American Phytopathological Society
The goals of this study were to determine the formae speciales of S. turcica in China and to analyze the relationships among genetic diversity, geographic distribution, and pathogenic specialization.

\section{Materials and Methods}

Purification and cultivation of pathogenic isolates. The isolates studied here were obtained using a tissue separation method (14) from samples of infected leaves of maize and sorghum collected from various regions of China. Monosporic cultures of $S$. turcica isolates were cultivated on potato dextrose agar (PDA; $200 \mathrm{~g}$ of potato, $20 \mathrm{~g}$ of glucose, $18 \mathrm{~g}$ of agar, and 1 liter of water) at $25^{\circ} \mathrm{C}$.

DNA extraction and identification of internal transcribed spacer regions. Each isolate was cultivated for 7 days on potato dextrose broth (PDB; $200 \mathrm{~g}$ of potato extract, $20 \mathrm{~g}$ of glucose, and 1 liter of water) at $25^{\circ} \mathrm{C}$. The mycelia in PDB were filtered with the double layer of sterile gauze, washed with sterile distilled water, and dried using FreeZone 2.5-liter Freeze Dry Systems (Light Ace HK TLD, Beijing) until they were completely dry (38). The dried mycelia were placed in liquid nitrogen in a mortar and ground with a pestle into a fine powder. Total DNA was extracted using an Ezup Column Fungi Genomic DNA Purification Kit (Sangon Biotech, Shanghai, China). DNA was quantified by measuring optical density at 260 or $280 \mathrm{~nm}$ (33) and using 1.2\% agarose gel electrophoresis. Experimental isolates were identified by comparing their internal transcribed spacer (ITS) sequence with that of an S. turcica reference isolate (National Center for Biotechnology Information [NCBI] accession number KF278460.1) maintained in our laboratory. ITS reactions $(50 \mu \mathrm{l})$ contained $5 \mu \mathrm{l}$ of $10 \times$ buffer, $3 \mu \mathrm{l}$ of $25 \mathrm{mM} \mathrm{MgCl} 2,4 \mu \mathrm{l}$ of $2.5 \mathrm{mM}$ dNTP, $1 \mu \mathrm{l}$ of $10 \mathrm{mM}$ ITS1 primer $(16,17), 1 \mu 1$ of $10 \mathrm{mM}$ ITS4 primer $(16,17)$,

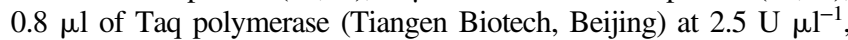
$1 \mu \mathrm{l}$ of $100 \mathrm{ng}$ of DNA, and $34.2 \mu \mathrm{l}$ of double-distilled $(\mathrm{dd}) \mathrm{H}_{2} \mathrm{O}$. The PCR program was as follows: $5 \mathrm{~min}$ at $94^{\circ} \mathrm{C}$; followed by 35 cycles at $94^{\circ} \mathrm{C}$ for $40 \mathrm{~s}, 56^{\circ} \mathrm{C}$ for $40 \mathrm{~s}$, and $72^{\circ} \mathrm{C}$ for $1 \mathrm{~min}$, with final extension at $72^{\circ} \mathrm{C}$ for $10 \mathrm{~min}(38)$. The amplified fragments were separated through a $1.5 \%$ agarose gel electrophoresis containing $1 \times$ Tris-borate-EDTA (TBE). The $1.5 \%$ agarose gel containing Goldview (Tiangen Biotech), which is a new and safe nucleic acid dye compared with ethidium bromide, was prepared $30 \mathrm{~min}$ before electrophoresis for $40 \mathrm{~min}$ at $110 \mathrm{~V}$. The gel was visualized to confirm the reproducibility of the band patterns and photographed using a UV Transilluminator (UVItec, Cambridge, UK). Nucleotide sequencing was performed by Sangon Biotech.

Pathogenicity tests. Susceptible cultivars were as follows: 'Huangzaosi' maize (lacking a resistance gene), 'LR115' sorghum, and 'Yuantai023' 
Table 1. Setosphaeria turcica isolates used for universally primed polymerase chain reaction and their accession numbers

\begin{tabular}{|c|c|}
\hline Strains & Accession numbers \\
\hline CCG1326 & KJ922714.1 \\
\hline SHG1303 & KJ922737.1 \\
\hline PLG1306 & KJ922734.1 \\
\hline JPG1302 & KJ922726.1 \\
\hline SYG1306 & KJ922739.1 \\
\hline YYG1302 & KJ922750.1 \\
\hline ZMDG1308 & KJ922752.1 \\
\hline YCG1301 & KJ922748.1 \\
\hline LLG1302 & KJ922728.1 \\
\hline XZG1312 & KJ922746.1 \\
\hline CZG1304 & KJ922718.1 \\
\hline HSG1302 & KJ922723.1 \\
\hline TLG1303 & KJ922741.1 \\
\hline LPG1304 & KJ922730.1 \\
\hline XAG1303 & KJ922744.1 \\
\hline MHKG1309 & KJ922732.1 \\
\hline DQG1301 & KJ922721.1 \\
\hline SYY1303 & KJ922740.1 \\
\hline JPY1302 & KJ922727.1 \\
\hline DLY1308 & KJ922720.1 \\
\hline DQY1306 & KJ922722.1 \\
\hline SHY 1309 & KJ922738.1 \\
\hline CCY1303 & KJ922715.1 \\
\hline MHKY1302 & KJ922733.1 \\
\hline PLY1305 & KJ922735.1 \\
\hline YCY1324 & KJ922749.1 \\
\hline YYY1319 & KJ922751.1 \\
\hline ZMDY1311 & KJ922753.1 \\
\hline LLY1305 & KJ922729.1 \\
\hline XZY1312 & KJ922747.1 \\
\hline CZY1321 & KJ922719.1 \\
\hline HSY1309 & KJ922724.1 \\
\hline TLY1308 & KJ922742.1 \\
\hline LPY1313 & KJ922731.1 \\
\hline XAY1302 & KJ922745.1 \\
\hline JNY1315 & KJ922725.1 \\
\hline QDY1307 & KJ922736.1 \\
\hline CDY1316 & KJ922716.1 \\
\hline CQY1310 & KJ922717.1 \\
\hline WQY1308 & KJ922743.1 \\
\hline
\end{tabular}

Sudan grass. The plants were grown in a greenhouse in plastic pots (three plants per pot) to the six-leaf stage before inoculation. Conidia were washed from 10-day-old pure cultures grown on PDA at $25^{\circ} \mathrm{C}$ using sterile distilled water. Conidial suspensions $\left(1 \times 10^{6}\right.$ conidial $/ \mathrm{ml}$ ) were prepared using sterile distilled water. Tween-20 was added into the suspension to a final concentration of $0.02 \%$. Each conidial suspension was used to inoculate maize, sorghum, and Sudan grass at the same time. To keep the leaf blades wet, plants were incubated at 20 to $26^{\circ} \mathrm{C}$ for $48 \mathrm{~h}$ in a plastic tent. The experimental design was completely randomized using three biological replicates. The plants were examined for symptoms of disease after 15 days and compared with those of corn northern leaf blight (Centro Internacional de Mejoramientode Maizy Trigo), which served as a positive control.

UP-PCR analysis. In all, 23 isolates of S. turcica. f. sp. zeae and 17 of $S$. turcica. f. sp. sorghi were selected as representatives of each forma specialis (Table 1). The UP primers AA2M2, AS15inv, AS4, AS15, HE45, L45, L15, L15/AS19, L21, 0.3-1, 0.3-2, 3-1, and 3-2 (32) were used to amplify the total DNA of each. PCR reactions were performed in a final volume of $20 \mu \mathrm{l}$ and contained $2 \mu \mathrm{l}$ of $10 \times$ buffer, $2 \mu \mathrm{l}$ of $25 \mathrm{mM} \mathrm{MgCl} 2,1.6 \mu \mathrm{l}$ of $2.5 \mathrm{mM}$ dNTP, $1 \mu \mathrm{l}$ of $10 \mathrm{mM}$ UP primer, $0.6 \mu \mathrm{l}$ of Taq polymerase (Tiangen Biotech) at

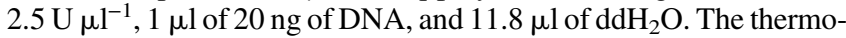
cycle programs was as follows: denaturation for $2 \mathrm{~min}$ at $94^{\circ} \mathrm{C}, 40 \mathrm{~s}$ at $53^{\circ} \mathrm{C}$, and $30 \mathrm{~s}$ at $72^{\circ} \mathrm{C}$; followed by 30 cycles at $92^{\circ} \mathrm{C}$ for $20 \mathrm{~s}$, $53^{\circ} \mathrm{C}$ for $40 \mathrm{~s}$, and $72^{\circ} \mathrm{C}$ for $30 \mathrm{~s}$; and final extension at $72^{\circ} \mathrm{C}$ for 2 min (18). PCR-amplified fragments were separated on a $1.5 \%$ agarose gel electrophoresis containing $1 \times$ TBE. The $1.5 \%$ agarose gel containing Goldview (Sangon Biotech) was prepared $30 \mathrm{~min}$ before electrophoresis for $40 \mathrm{~min}$ at $110 \mathrm{~V}$. The gel was visualized to confirm the reproducibility of the bands patterns and photographed using a UV transilluminator (UVItec).

Data analysis. Amplified bands generated using each primer set were assigned as present $=1$ or absent $=0$, followed by cluster analysis using the unweighted pair group method with arithmetic mean (UPGMA) (31) implemented in the NTSYS-pc program, version $2.02(32)$.

\section{Results}

Morphological studies and identification of ITS sequences. Colony morphology, hyphae, conidiophores, and conidia of 188 isolates of $S$. turcica collected in China were examined in the study. Colonies were ash black and circular with gray aerial hyphae. Brown

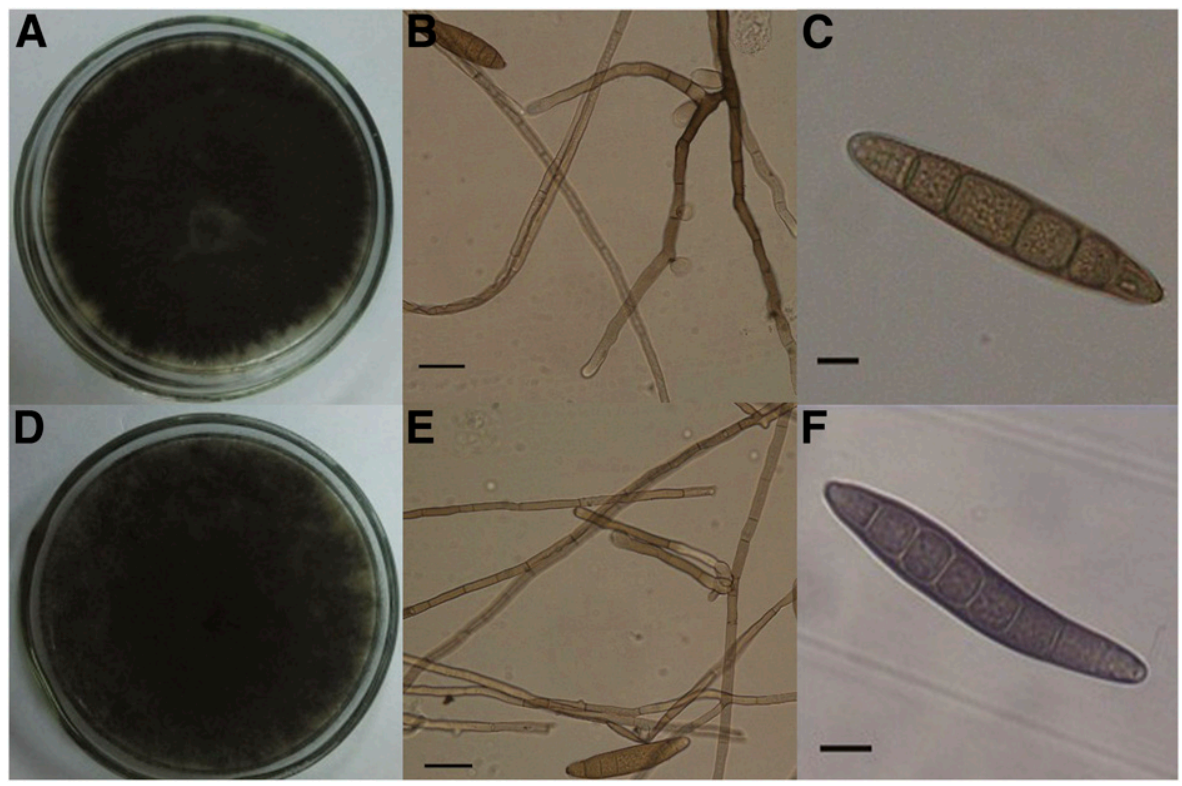

Fig. 1. Morphology of Setosphaeria turcica from maize (isolate SYY1326): A, colony morphology; B, hyphae and conidiophores morphology; C, conidial morphology. Morphology of S. turcica from sorghum (strain JPG1306): D, colony morphology; $E$, hyphae morphology; $F$, conidial morphology. Bars: $B$ and $E=20 \mu \mathrm{m}, C$ and $F=10 \mu \mathrm{m}$. 
filiform conidiophores were erect or flexuous, single or tufty, with larger basal cells, light brown tops, and two to six cross septa, and they were 32.6 to $180 \mu \mathrm{m}$ long and 5.6 to $13.1 \mu \mathrm{m}$ wide. Conidia were olive-brown, long, and spindle-shaped, with long elliptical apical cells, pointed cone basal cells, and obvious umbilic points outside the basal cell with two to eight cross septa, and they were 48.7 to $126.9 \mu \mathrm{m}$ long and 11.2 to $23.6 \mu \mathrm{m}$ wide. The morphology, colony size, hyphae, conidiophores, and conidia were similar among all isolates (Fig. 1). There were no differences in morphology among $S$. turcica isolates derived from different hosts. There was as much as $99 \%$

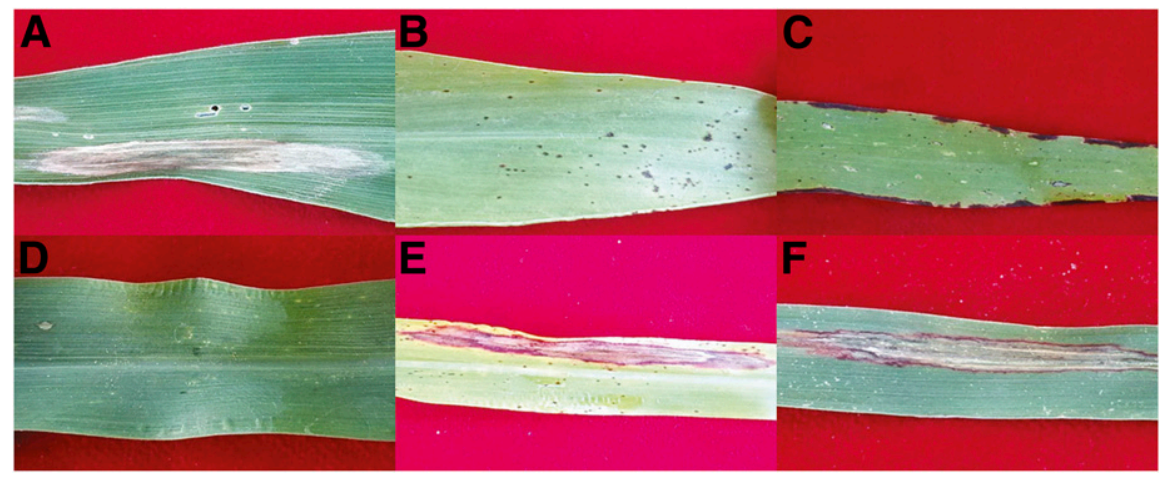

Fig. 2. Pathogenicity tests of Setosphaeria turcica on maize and sorghum. A, Typical disease spot of $S$. turcica. f. sp. zeae on maize; B, no visible reaction of S. turcica. f. sp. zeae on sorghum except some flecks; C, no visible reaction of $S$. turcica. f. sp. zeae on Sudan grass except some flecks; D, typical disease spot of $S$. turcica. f. sp. sorghi on sorghum; E, typical disease symptom of $S$. turcica. f. sp. sorghi on Sudan grass; F, no visible reaction of $S$. turcica. f. sp. sorghi on maize.

Table 2. Isolates of Setosphaeria turcica included in the study, their formae specialis, and origins

\begin{tabular}{|c|c|c|}
\hline Formae specialis, strains ${ }^{\mathbf{a}}$ & Numbers & Geographic origin $^{b}$ \\
\hline \multicolumn{3}{|l|}{ S. turcica. f. sp. sorghi } \\
\hline $\begin{array}{l}\text { PLG1301, PLG1302, PLG1303, } \\
\text { PLG1304,PLG1305, PLG1306* }\end{array}$ & 6 & Pingliang, Gansu \\
\hline DQG1301*, DQG1302, DQG1303 & 3 & Daqing, Helongjiang \\
\hline HGG1301, HGG1302 & 2 & Hegang, Helongjiang \\
\hline SHG1303*, SHG1311 & 2 & Suihua, Helongjiang \\
\hline $\begin{array}{l}\text { JPG1302*, JPG1304, JPG1305, } \\
\text { JPG1306 }\end{array}$ & 4 & Jianping, Liaoning \\
\hline FCG1303, FCG1305, FCG1307 & 3 & Fengcheng, Liaoning \\
\hline $\begin{array}{l}\text { SYG1302, SYG1303, SYG1305, } \\
\text { SYG1306*,SYG1307, SYG1310, } \\
\text { SYG1311, SYG1312, SYG1317 }\end{array}$ & 9 & Shenyang, Liaoning \\
\hline LLG1302*, LLG1321, LLG1323 & 3 & Lvliang, Shanxi \\
\hline XZG1308, XZG1312* & 2 & Xinzhou, Shanxi \\
\hline CZG1304*, CZG1307, CZG1318 & 3 & Cangzhou, Hebei \\
\hline HSG1301, HSG1302* & 2 & Hengshui, Hebei \\
\hline TLG1302, TLG1303*, TLG1306 & 3 & Tongliao, Neimeng \\
\hline $\begin{array}{l}\text { LPG1301, LPG1302, LPG1303, } \\
\text { LPG1304*, LPG1305 }\end{array}$ & 5 & Luoping, Yunnan \\
\hline $\begin{array}{l}\text { XAG1301, XAG1302, XAG1303*, } \\
\text { XAG1304, XAG1305 }\end{array}$ & 5 & Xi'an, Shaanxi \\
\hline YLG1301, YLG1302, YLG1307 & 3 & Yangling, Shaanxi \\
\hline YCG1301*, YCG1302, YCG1305 & 3 & Yichuan, Henan \\
\hline $\begin{array}{l}\text { LYG1301, LYG1303, LYG1306, } \\
\text { LYG1309 }\end{array}$ & 4 & Luoyang, Henan \\
\hline $\begin{array}{l}\text { ZMDG1308*,ZMDG1311, } \\
\text { ZMDG1322 }\end{array}$ & 3 & Zumadian, Henan \\
\hline YYG1302*, YYG1304, YYG1305 & 3 & Yuanyang, Henan \\
\hline $\begin{array}{l}\text { MHKG1302, MHKG1304, } \\
\text { MHKG1309*, MHKG1314 }\end{array}$ & 4 & Meihekou, Jilin \\
\hline CCG1312, CCG1317, CCG1326* & 3 & Changchun, Jilin \\
\hline \multicolumn{3}{|l|}{ S. turcica. f. sp. zeae } \\
\hline $\begin{array}{l}\text { SYY1301, SYY1303*, SYY1306, } \\
\text { SYY1326 }\end{array}$ & 4 & Shenyang, Liaoning \\
\hline TLY1302, TLY1305, TLY1311 & 3 & Tieling, Liaoning \\
\hline JPY1301, JPY1302*, JPY1307 & 3 & Jianping, Liaoning \\
\hline DLY1308*, DLY1316 & 2 & Dalian, Liaoning \\
\hline DDY1321 & 1 & Dandong, Liaoning \\
\hline \multicolumn{3}{|c|}{ (continued in next column) } \\
\hline
\end{tabular}

Table 2. (continued from preceding column)

\begin{tabular}{|c|c|c|}
\hline Formae specialis, strains $^{\mathbf{a}}$ & Numbers & Geographic origin $^{b}$ \\
\hline WFDY1314, WFDY1332 & 2 & $\begin{array}{l}\text { Wangfangdian, } \\
\text { Liaoning }\end{array}$ \\
\hline $\begin{array}{l}\text { FCY1303, FCY1304, FCY1309, } \\
\text { FCY1311 }\end{array}$ & 4 & Fengcheng, Liaoning \\
\hline $\begin{array}{l}\text { HEBY1304, HEBY1307, } \\
\text { HEBY1310, HEBY1311 }\end{array}$ & 4 & Habin, Helongjiang \\
\hline $\begin{array}{l}\text { DQY1301, DQY1305, DQY1306*, } \\
\text { DQY1309, DQY1321 }\end{array}$ & 5 & Daqing, Heilongjiang \\
\hline SHY1303, SHY1309*, SHY1313 & 3 & Suihua, Helongjiang \\
\hline $\begin{array}{l}\text { HGY1308, HGY1311, HGY1313, } \\
\text { HGY1315 }\end{array}$ & 4 & Hegang, Helongjiang \\
\hline CCY1302, CCY1303*, CCY1309 & 3 & Changchun, Jiling \\
\hline $\begin{array}{l}\text { MHKY1301, MHKY1302*, } \\
\text { MHKY1311, MHKY1320, } \\
\text { MHKY1321 }\end{array}$ & 5 & Meihekou, Jilin \\
\hline $\begin{array}{l}\text { SPY1302, SPY1303, SPY1305, } \\
\quad \text { SPY1306 }\end{array}$ & 4 & Siping, Jilin \\
\hline PLY1301, PLY1302, PLY1305* & 3 & Pingliang, Gansu \\
\hline $\begin{array}{l}\text { LZY1302, LZY1304, LZY1305, } \\
\text { LZY1312 }\end{array}$ & 4 & Lanzhou, Gansu \\
\hline QDY1305, QDY1307*, QDY1309 & 3 & Qingdao, Shandong \\
\hline JNY1314, JNY1315*, JNY1318 & 3 & Jinan, Shandong \\
\hline CDY1314, CDY1315, CDY1316* & 3 & Chengdu, Sichuan \\
\hline $\begin{array}{l}\text { CQY1305, CQY1307, CQY1309, } \\
\text { CQY1310* }\end{array}$ & 4 & Congqing \\
\hline $\begin{array}{l}\text { LYY1301, LYY1303, LYY1304, } \\
\text { LYY1306 }\end{array}$ & 4 & Luoyang, Henan \\
\hline YCY1305, YCY1312, YCY1324* & 3 & Yichuan, Henan \\
\hline YYY1314, YYY1315, YYY1319* & 3 & Yuanyang, Henan \\
\hline $\begin{array}{l}\text { ZMDY1308, ZMDY1311*, } \\
\text { ZMDY1319 }\end{array}$ & 3 & Zhumadian, Henan \\
\hline TYY1302, TYY1307, TYY1311 & 3 & Taiyuan, Shanxi \\
\hline LLY1303, LLY1305* & 2 & Lvliang, Shanxi \\
\hline XZY1311, XZY1312*, XZY1315 & 3 & Xinzhou, Shanxi \\
\hline CZY1301, CZY1313, CZY1321* & 3 & Cangzhou, Hebei \\
\hline HSY1304, HSY1307, HSY1309* & 3 & Hengshui, Hebei \\
\hline $\begin{array}{l}\text { TLY1306, TLY1308*, TLY1309, } \\
\text { TLY1310, TLY1312, TLY1314 }\end{array}$ & 6 & Tongliao, Neimeng \\
\hline LPY1307, LPY1309, LPY1313* & 3 & Luoping, Yunnan \\
\hline $\begin{array}{l}\text { XAY1301, XAY1302*, XAY1303, } \\
\text { XAY1309 }\end{array}$ & 4 & Xi'an, Shaanxi \\
\hline YLY1306, YLY1310, YLY1316 & 3 & Yangling, Shaanxi \\
\hline WQY1308*,WQY1310, WQY1322 & 3 & Wuqing, Tianjin \\
\hline Sum & 188 & $\ldots$ \\
\hline
\end{tabular}




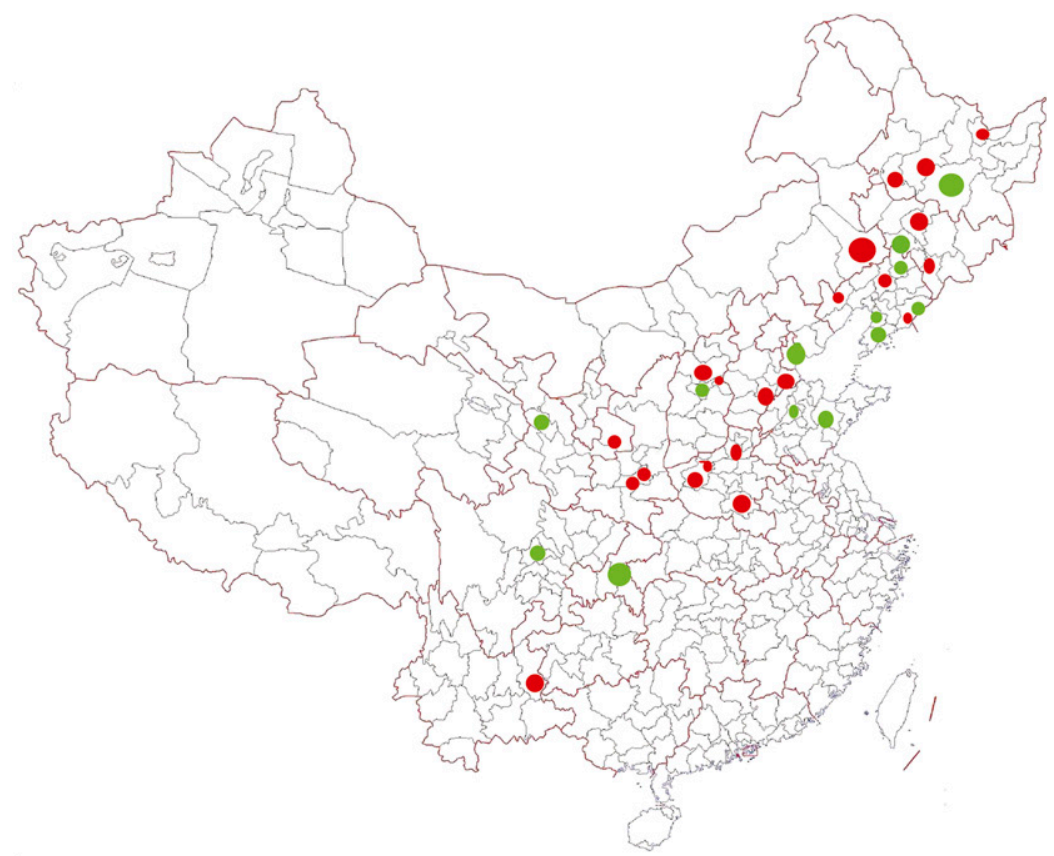

Fig. 3. Geographic distributions of the formae specialis of Setosphaeria turcica. Red areas are common geographic distributions of S. turcica. f. sp. sorghi and S. turcica. f. sp. zeae, and green areas are geographic distributions of S. turcica. f. sp. zeae but no S. turcica. f. sp. sorghi.

Table 3. Primers, their sequence and number of bands scored in universally primed polymerase chain reaction UP-PCR analysis of Setosphaeria turcica.

\begin{tabular}{llcccc}
\hline Primers & \multicolumn{1}{c}{ Nucleotide sequence 5' $\mathbf{- 3}^{\prime}$} & Scored bands & Polymorphic bands & Homology radio (\%) & Polymorphism Radio (\%) \\
\hline L21 & GGATCCGAGGGTGGCGGTTCT & 13 & 10 & $\ldots$ & $\ldots$ \\
HE45 & GTAAAACGAGGCCAGT & 9 & 4 & $\ldots$ & $\ldots$ \\
$0.3-2$ & TGAGGACAACGGTTCT & 10 & 10 & $\ldots$ & $\ldots$ \\
AA2M2 & CTGCGACCCAGAGCGG & 14 & 12 & $\ldots$ & $\ldots$ \\
AS15inv & CATTGCTGGCGAATCGG & 16 & 15 & 17.7 & $\ldots$ \\
Total & $\ldots$ & 62 & 51 & & $\ldots$ \\
\hline
\end{tabular}

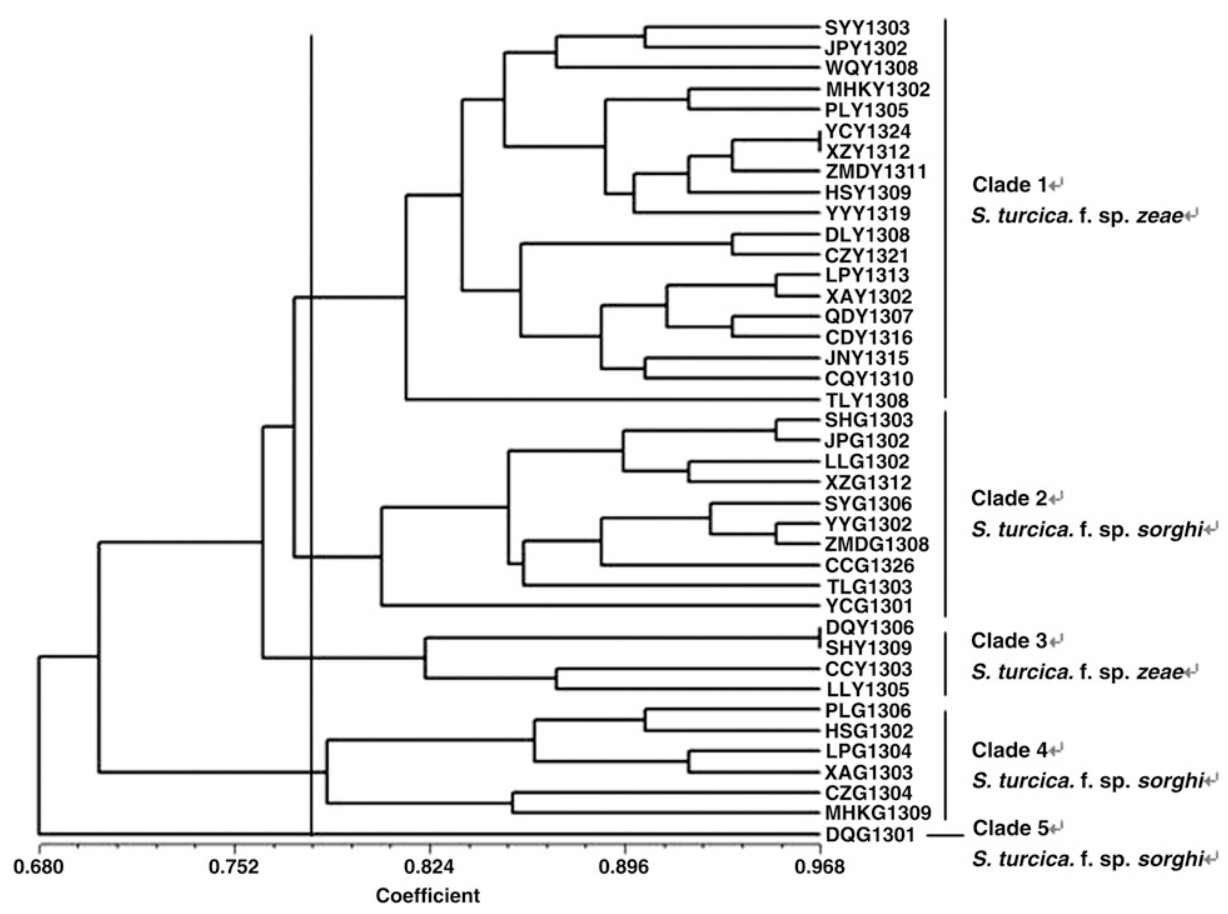

Fig. 4. Unweighted pair group method with arithmetic mean dendrogram of analyzed Setosphaeria turcica. f. sp. zeae and S. turcica. f. sp. sorghi isolates obtained from coefficient similarity matrix using universally primed polymerase chain reaction marker data. 
DNA sequence identity (the value of "sort by ident") compared with the reference isolate (NCBI accession number KF278460.1) among all isolates. Taken together, the results of these analyses indicate that all isolates were $S$. turcica.

Pathogenicity analysis. All 188 isolates revealed significant differences in virulence compared with the corn northern leaf blight control. Thus, maize blades were covered with fusiform necrotic spots after inoculation with isolates from maize, which had darkbrown centers and light-gray margins. The incompatible reactions observed on sorghum and Sudan grass were fuchsia-colored flecks (Fig. 2A to C). The long fusiform necrotic spots with light-brown centers and fuchsia-colored margins were formed on the blades of the sorghum and Sudan grass after inoculation with isolates from sorghum. Incompatible reactions appeared symptomless on maize (Fig. 2D and E). There were 113 S. turcica. f. sp. zeae and 75 S. turcica. f. sp. sorghi isolates among the 188 isolates (Table 2) but no isolates of $S$. turcica. f. sp. complexa.

Two formae speciales of $S$. turcica were widely dispersed among the regions where maize and sorghum are cultivated in China (Fig. 3; Table 2). S. turcica. f. sp. zeae and S. turcica. f. sp. sorghi were present in 34 regions of 14 provinces and in 21 regions of 10 provinces, respectively. Because sorghum is cultivated in smaller areas compared with maize, S. turcica. f. sp. sorghi was not isolated from plants in certain regions such as Shangdong province (Qingdao and Jinan), Sichuan province (Chengdu), Chongqing, Tianjin (Wuqing), Gansu province (Lanzhou), Liaoning province (Dalian, Tieling, Dandong, Wangfangdian), Jilin province (Siping), Heilongjiang province (Habin), and Shanxi province (Taiyuan). Therefore, pathogenicity did not directly correlate with the geographic distributions in all isolates.

UP-PCR analysis. In all, $17 \mathrm{~S}$. turcica. f. sp. sorghi isolates and 23 S. turcica. f. sp. zeae isolates were analyzed using UP-PCR. Primers L21, HE45, 0.3-2, AA2M2, and AS15inv generated polymorphic banding patterns and were used for UP-PCR analysis (Table 3). There were 62 amplified bands and 51 polymorphic bands. The homology to polymorphism ratios in the bands from the five UP-PCR primers was 17.7 and $82.3 \%$, respectively.

A dendrogram generated from the UPGMA analysis is shown in Figure 4 . The phenon line, representing the mean between similar isolates, was drawn at $78 \%$. At this level, the $40 \mathrm{~S}$. turcica isolates were divided into five clades. Clades 1 and 3 included $S$. turcica. f. sp. zeae. Clades 2, 4, and 5 included S. turcica. f. sp. sorghi. The isolates of each clade belonged to the same forma specialis, and different formae speciales were assigned to different clades. Consistent with the results of pathogenicity tests and findings that two formae speciales were widespread in the planting areas of maize and sorghum in China, the UPPCR analysis indicates a correlation between pathogenic specialization and genetic diversity. Therefore, UP-PCR is useful for the study of genetic relations among the formae speciales of $S$. turcica and reveals a high level of genetic diversity among these pathogens.

According to their geographic distribution, the same forma specialis from different provinces were present in as the same clade. For example, LLY1305, CCY1303, and SHY1309 were assigned to clade 3, although they were isolated from the provinces of Shanxi, Jilin, and Helongjiang, respectively. However, in some instances, the same forma specialis from the same province was classified in a different clade. For example, DQG1301 and SHG1303 were assigned to in clades 2 and 5, respectively, although they were isolated from Helongjiang province. Therefore, UP-PCR analysis did not indicate a correlation between and genetic diversity and geographic distribution.

\section{Discussion}

No differences in morphology were observed among the $S$. turcica strains derived from different hosts, which is consistent with previous reports $(1,3,4,13,20,21,25,26,29,30,34)$. The pathogenicity assays performed in the present study identified 113 S. turcica. f. sp. zeae isolates and $75 \mathrm{~S}$. turcica. f. sp. sorghi isolates but did not identify the $S$. turcica. f. sp. complexa among a collection of 188 isolates. Two formae speciales of $S$. turcica were widespread in regions in China where maize and sorghum are cultivated.
UP-PCR analysis demonstrated genetic differences among the formae speciales of $S$. turcica and divided them into five clades that include the same forma specialis and isolates of from the different provinces in the same clade and the same forma specialis of the same provinces in different clades. The results of pathogenicity assays and UP-PCR analysis indicated a closer correlation between pathogenic specialization and genetic diversity but not geographic distribution. These conclusions are consistent with those of other studies $(5,27,37)$.

Pathogenicity tests are influenced by variables such as temperature (35), age of the host (12), and the method of inoculation (19). Therefore, the goal of the present study was to develop a PCR-based method for studying genetic diversity of formae speciales of $S$. turcica isolates to better understand disease control, population dynamics, and epidemiology. The UP-PCR molecular marker was designed to identify genomic differences without previous knowledge of the target DNA sequences. These results were analyzed for their correlation with colony morphology (22), pathogenicity, geographic distribution (27), and formae speciales (2,11), as well as other factors (24). Therefore, the present study illustrates the utility of UP-PCR technology for supplementing pathogenicity tests to accurately characterize the genetic diversity and phylogenetic relationships among the formae speciales of $S$. turcica. However, because of the limited number of geographic regions studied here and the insufficient number of S. turcica. f. sp. complexa isolates, further research is required fully to characterize the formae speciales in $S$. turcica.

\section{Acknowledgments}

We thank X. Xu and Y. Jiang (Institute of Plant Protection, Liaoning Academy of Agricultural Sciences, Shenyang, China) for providing the S. turcica stains. This project was supported by the National Key Technology Research and Development Program of Program Program (number 2012BAD19B04) and Research Projects in Liaoning Province (number 2014201003).

\section{Literature Cited}

1. Ali, H. H., and Aragaki, M. 1974. Inheritance of pathogenicity in Setosphaeria turcica. Phytopathology 65:280-283.

2. Baayen, R. P., O’Donnell, K., Bonants, P. J. M., Cigelnik, E., Kroon, L. P., Roeroeck, E. J., and Waalwijk, C. 2000. Gene genealogies and AFLP analysis in the Fusarium oxysporum complex identify monophyletic and nonmonophyletic formae specialis causing wilt and rot diseases. Phytopathology 90: 891-900.

3. Bergquist, R. R., and Masias, O. R. 1974. Physiologic specialization in Trichometasphaeria turcica f. sp. zeae and T. turcica f. sp. sorghi in Hawaii. Phytopathology 64:645-649.

4. Bhowmik, T. P., and Prasada, R. 1970. Physiologic specialization in Helminthosporium turcicum Pass. from India. J. Phytopathol. 68:84-87.

5. Bogale, M. B., Wingfield, B. D., Wingfield, M. J., and Steenkamp, E. T. 2006. Characterization of Fusarium oxysporum strains from Ethiopia using AFLP, SSR and DNA sequence analysis. Fungal Divers. 23:51-66.

6. Bulat, S. A., Lübeck, M., Mironenko, N. V., Jensen, D. F., and Lübeck, P. S. 1998. UP-PCR analysis and ITS1 ribotyping of strains of Trichoderma and Gliocladium. Mycol. Res. 102:933-943.

7. Bulat, S. A., and Mironenko, N. V. 1990. Species identity of the phytopathogenic fungi Pyrenophora teres Dreschler and P. graminea Ito and Kuribayashi. (in Russian) Mikol. Fitopatol. 24:435-441.

8. Bulat, S. A., and Mironenko, N. V. 1996. Identification of fungi and analysis of their genetic diversity by polymerase chain reaction (PCR) with genespecific and nonspecific primers. Russ. J. Genet. 32:143-159.

9. Bulat, S. A., Mironenko, N. V., Lapteva, M. N., and Strelchenko, P. P. 1994 Polymerase chain reaction with universal primers (UP-PCR) and its application to plant genome analysis. Pages 113-129 in: Conservation of Plant Genes II: Utilization of Ancient and Modern DNA. R. P. Adams, J. S. Miller, E. M. Goldenberg, and J. E. Adams, eds. Missouri Botanical Garden, St. Louis.

10. Caligiorne, R. B., Resende, M. A., Diasneto, E., Oliveira, S. C., and Azevedo, V. 1999. Dematiaceous fungal pathogens: analysis of ribosomal DNA gene polymorphism by polymerase chain reaction - restriction fragment length polymorphism. Mycoses 42:609-614.

11. Chang, H. S., and Fan, K. C. 1986. Comparative studies on some biology and pathology of corn and broom corn strains of Exserohilum turcicum (Pass.) Leonard \& Suggs. Bot. Bull. Acad. Sin. 27:209-218.

12. Correll, J. C. 1991. The relationship between formae speciales, races, and vegetative compatibility groups in Fusarium oxysporum. Phytopathology 81:1061-1064.

13. Elad, Y., Chet, I., Boyle, P., and Henis, Y. 1983. Parasitism of Trichoderma sp. on Rhizoctonia solani and Sclerotium rolfsii. Scanning electron microscopy and fluorescence microscopy. Phytopathology 73:85-88. 
14. Fang, Z. D. 1998. Isolation and cultivation of fungi. Pages 125-137 in: Methods in Plant Pathology, 3rd ed. China Agriculture Press, Beijing.

15. Gao, J. X., Gao, Z. G., and Lv, S. X. 2011. Establishment of UP-PCR system and genetic diversity analysis of Exserohilum turcicum. J. Maize Sci. 19:112-117.

16. Gardes, M., and Bruns, P. B. 1993. ITS primers with enhanced specificity of Basidiomycetes: Application to the identification of mycorrhizae and rusts. Mol. Ecol. 2:113-118.

17. Gordon, T. R., and Okamoto, D. 1991. Vegetative compatibility groupings in a local population of Fusarium oxysporum. Can. J. Bot. 69:168-172.

18. Hammerschmidt, R., Metraux, J. P., and Van Loon, L. C. 2001. Inducing resistance: A summary of papers presented at the first international symposium on induced resistance to plant diseases, Corfu, May 2000. Eur. J. Plant Pathol. 107:1-6.

19. Kraft, J. M., and Haglund, W. A. 1978. A reappraisal of the race classification of Fusarium oxysporum f. sp. pisi. Phytopathology 68:273-275.

20. Lefebvre, C. L., and Sherwin, H. S. 1945. Races of Helminthosporium turcicum. (Abstr.) Phytopathology 35:487.

21. Leonard, K. J., and Suggs, E. G. 1974. Setosphaeria prolata, the ascigerous state of Exserohilum prolatum. Mycologia 66:281-297.

22. Longato, S., and Bonfante, P. 1997. Molecular identification of mycorrhizal fungi by direct amplification of microsatellite regions. Mycol. Res. 101: 425-432.

23. Lubeck, M., and Poulsen, H. 2001. UP-PCR cross blot hybridization as a tool for identification of anastomosis groups in the Rhizoctonia solani complex. FEMS Microbiol. Lett. 201:83-89.

24. Marlatt, M. L., Correll, J. C., Kaufmann, P., and Cooper, P. E. 1996. Two genetically distinct populations of Fusarium oxysporum f. sp. lycopersici race 3 in the United States. Plant Dis. 80:1336-1342.

25. Masias, O. R., and Bergouist, R. R. 1974. Host specific forms of Trichometasphaeria turcica in relation to homokaryons and heterokaryons in nature. Phytopathology 64:436-438.

26. Mitra M. 1923. Helminthosporium spp. on cereals and sugarcane in India. Diseases of Zea mays and Sorghum vulgare caused by species of Helminthosporium. Part I. India Dep. Agric. Mem. Bot. Ser. 11:219-242.
27. Muiru, W. M., Koopmann, B., Tiedemann, A. V., Mutitu, E. W., and Kimenju, J. W. 2010. Evaluation of genetic variability of Kenyan, German and Austrian isolates of Exserohilum turcicum using Amplified fragment length polymorphism DNA marker. Biotechnology 9:204-211.

28. Naumov, G. I., Naumova, E. S., Kondratieva, V. I., Bulat, S. A., Mironenko, N. V., Mendonca-Hagler, L. C., and Hagler, A. N. 1997. Genetic and molecular delineation of three sibling species in the Hansenula polymorpha complex. Syst. Appl. Microbiol. 20:50-56.

29. Robert, A. L. 1960. Physiologic specialization in Helminthosporium turcicum. Phytopathology 50:217-220.

30. Rodriguez, A. E., and Ullstrup, A. J. 1962. Pathogenicity of monoascosporic strains of Trichometasphaeria turcica. Phytopathology 52:599-601.

31. Rohlf, F. J.1993. NTSYS-pc: Numerical Taxonomy and Multivariate Analysis System. Exeter Publishing Ltd., New York.

32. Rohlf, F. J. 1997. NTSYS-pc: Numerical Taxonomy and Multivariate Analysis System, Version 2.02. Exeter Software, Setauket, NY.

33. Sambrook, J. 1989. Molecular Cloning: A Laboratory Manual, 2nd ed. Cold Spring Harbor Laboratory Press, Cold Spring Harbor, NY.

34. Tarr, S. A. J. 1962. Disease of sorghum Sudan grass and broom corn. Ph.D thesis, The Commonwealth Mycological Institute, University of Edinburgh, Kew, Surrey, UK

35. Williams, P. H. 1981. Pages 124-129 in: Fusarium Yellows, Screening Crucifers for Multiple Disease Resistance. University of Wisconsin, Madison.

36. Yli-Mattila, T., Mach, R. L., Alekhina, A., Bulat, S. A., and Koskinen, S. 2004. Phylogenetic relationship of Fusarium langsethiae to Fusarium poae and Fusarium sporotrichioides as inferred by IGS, ITS, $\beta$-tubulin sequences and UP-PCR hybridization analysis. Int. J. Food Microbiol. 95: 267-285.

37. Zhang, S., Zhao, B. X., Liu, X., Li, J. B., Gao, Z. G., and Huang, X. Y. 2013 DNA Sequencing and UP-PCR Characterization of Fusarium oxysporum isolates from three cucurbit species. Plant Pathol. J. 12:78-84.

38. Zhao, H. 2009. The Physiological Differentiation and the Genetic Polymorphism of Exserohilum turcicum. Ph.D. Thesis, Shenyang agricultural university, Shenyang, China. 